\title{
ERRATUM
}

G. Urios • A. Martínez-Abraín

\section{The study of nest-site preferences in Eleonora's falcon Falco eleonorae through digital terrain models on a western Mediterranean island}

Published online: 6 April 2006

(C) Dt. Ornithologen-Gesellschaft e.V. 2006

\section{J Ornithol (2005). DOI: 10.1007/s10336-005-0097-2}

Unfortunately, the publisher printed the caption to Fig. 2 incorrectly. The correct caption is given here:

Fig. 2 Elevation digital terrain model

The online version of the article can be found at http://dx.doi.org/ 10.1007/s10336-005-0097-2

G. Urios $(\bowtie)$

Department de Biología Vegetal, Universitat de València,

C/Dr. Moliner 50, 46100 Burjassot, Valencia, Spain

E-mail: guriol01@tiscali.es

Tel.: + 34-96-5567232

A. Martínez-Abraín

IMEDEA (CSIC-UIB), C/Miquel Marqués 21 Majorca,

07190 Esporles, Spain

Present address: G. Urios

Plaza del Carmen 6-3 46003 Valencia, Spain 